\title{
Dual-Dipole UHF RFID Tag Antenna with Quasi-Isotropic Patterns Based on Four-Axis Reflection Symmetry
}

\author{
Chunfang Qin, ${ }^{1,2}$ Lingfei Mo, ${ }^{3}$ Hongliang Zhou, ${ }^{1}$ and Hongjian Zhang ${ }^{1}$ \\ ${ }^{1}$ State Key Laboratory of Industrial Control Technology, Department of Control Science and Engineering, Zhejiang University, \\ Hangzhou 310027, China \\ ${ }^{2}$ College of Information Science and Engineering, Guangxi University for Nationalities, Nanning 530006, China \\ ${ }^{3}$ School of Instrument Science and Engineering, Southeast University, Nanjing 210096, China
}

Correspondence should be addressed to Chunfang Qin; qinchf@gmail.com

Received 30 January 2013; Revised 3 April 2013; Accepted 7 April 2013

Academic Editor: Guo-Ping Gao

Copyright (C) 2013 Chunfang Qin et al. This is an open access article distributed under the Creative Commons Attribution License, which permits unrestricted use, distribution, and reproduction in any medium, provided the original work is properly cited.

In many RFID practical applications, it is required that reader can effectively read tags which are placed in radiation covering area randomly. In this paper, a passive UHF dual-dipole tag antenna with quasi-isotropic patterns is designed, which can reduce the sensibility of tag read-orientation in a long distance. Two dipoles with four-axis reflection symmetric structure are used, and the two arms of the dipole are bent to fill the space of the antenna. In this way, a quasi-isotropic tag is easier to be obtained. The test results show that the gain deviation of the proposed antenna was less than $3.25 \mathrm{~dB}$, and the maximum reading range in different directions was from $6.9 \mathrm{~m}$ to $10.0 \mathrm{~m}$, with better quasi-isotropic performance and reading range than other commercial tags.

\section{Introduction}

Radio frequency identification (RFID) technology is a noncontact automatic identification technique that acquires relevant data of an object with radio frequency to identify it. Since the passive ultra-high frequency (UHF) technology is characterized by great identification speed, multitarget identification, and long identification distance, it has been widely used in logistics and supply management, product manufacture and installation, library management, transportation, and other fields. A UHF RFID system consists of two units, a reader and a tag. Since a tag is not always oriented to a reader in some practical applications, such as management for luggage in airport or garment retail, it is required that reader can read tags which are at random placed within radiation coverage. Ordinary tag is usually a passive single-dipole tag and has a null reading zone along its axis, which may cause missing a read.

Isotropic antenna is a hypothetical antenna that radiates energy equally in all directions of the space. In reality, an antenna whose gain deviation in any arbitrary direction is less than $6 \mathrm{~dB}$ can be considered as a quasi-isotropic antenna
[1]. In recent years, some scholars have studied the quasiisotropic tag antennas. Kholodnyak et al. designed a threedimensional antenna to eliminate the sensibility of tag in reading orientation $[2,3]$. However, for its three-dimensional structure, the antenna is inconvenient for manufacture and can only be used in some special cases. Ahn et al. introduced some planar tags that are insensitive to the reading orientations $[1,4-8]$. These tags are designed on the basis of single-port chips which can be easily obtained from the market with low cost, while the reading range of the tags is only about two meters. But for logistics or other applications, the tag with a larger reading range is more practical. There are three reasons: firstly, in some passages for large cargo, a tag is required to be read from more than three meters; secondly, reading distance in the air will be shortened when a number of tags are stacked; and thirdly, the tag with a larger reading range is more practical and suitable for various applications. Therefore, it is important to design a quasi-isotropic tag not only with larger reading range but also with less directional sensitivity. There are also some commercial quasi-isotropic tags based on the dual-dipole antennas, which have large reading ranges, such as Invengo 


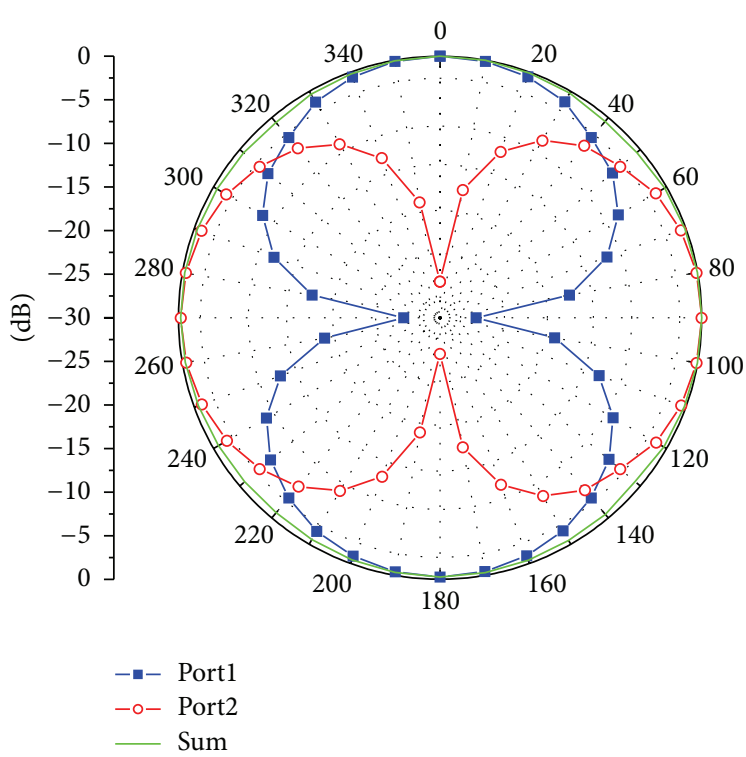

(a) Four-axis reflection symmetry

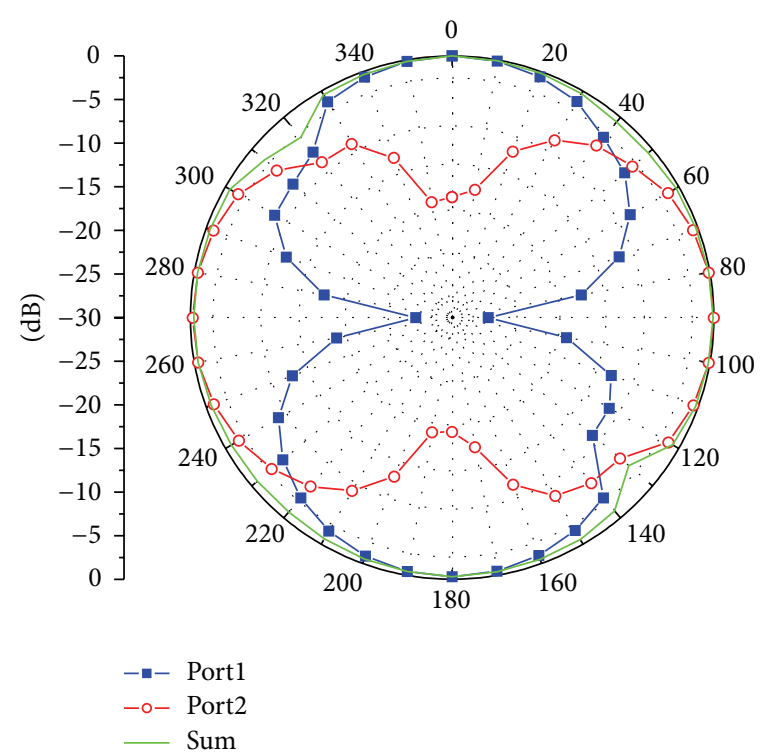

(b) Non-four-axis reflection symmetry

FIGURE 1: Radiation patterns of tag antenna with four-axis reflection symmetry and non-four-axis reflection symmetry.

TF8023, Impinj H47, and UPM frog 3D. However, some measurement results show that the gain deviations of the above quasi-isotropic dual-dipole tags are always greater than $5 \mathrm{~dB}$. Actually, with appropriate design for the tag antenna, its performance of quasi-isotropic patterns can be further improved.

In this paper, a passive UHF dual-dipole tag antenna with quasi-isotropic patterns is designed. Two dipoles with fouraxis reflection symmetric structure are adopted; namely, after being folded the tag antenna can completely overlap not only along the axis of $0^{\circ}-180^{\circ}$ or $90^{\circ}-270^{\circ}$, but also along the axis of $45^{\circ}-225^{\circ}$ or $135^{\circ}-315^{\circ}$. The two arms of the dipole are folded to fill the space of the antenna. If only the resonant frequency of this antenna is adjusted in an appropriate range, its gain deviation in any arbitrary direction in the space can be held to be about $3 \mathrm{~dB}$ and a quasi-isotropic tag is easier to be implemented. The test results show that the gain deviation of the tag designed in this paper could achieve less than $3.25 \mathrm{~dB}$ and the maximum reading range in different directions was up to $6.9-10.0$ meters.

The rest of this paper is organized as follows. In Section 2, the design of the antenna with four-axis reflection symmetry is discussed. In Section 3, the proposed antenna and three commercial tag antennas are simulated. Section 4 shows the measurement and analysis of the antennas. Section 5 is the conclusion.

\section{Design of the Antenna with Four-Axis Reflection Symmetry}

Ordinary dipole tag is generally thin and long and is a deformation of half-wave dipole. The antenna is fed from its center. Hence its electric current is distributed along the antenna as roughly sinusoidal pattern and is zero at both ends. If the axis of an ordinary dipole tag coincides with $Y$ axis in coordinate, the radiation pattern of the dipole tag in $X Z$ plane is uniform, but the shape of radiation pattern in $Y Z$ plane and $X Y$ plane is like an " 8 ." A reader can hardly read a tag if it points at the null reading zone for the tag along its axis. The gain deviation of an ordinary dipole in the space is generally $25-50 \mathrm{~dB}$.

To weaken the influence of null reading zone of an ordinary dipole and guarantee a large reading range, a kind of dual-dipole that consists of two mutually perpendicular dipoles can be designed. The two dipoles are axial symmetric to offset respective null reading zone of a single dipole. For a half-wave dipole, its half power beam width is about $80^{\circ}$, so two mutually perpendicular dipoles can cover a half power band width of about $320^{\circ}$ in the whole space. And in the rest space of about $40^{\circ}$, the power radiated by the two dipoles will be added, so the gain along the axis of two antennas will greatly increase to offset null reading zone of a single dipole. If the dual-dipole tag antenna designed in this paper completely overlaps after being folded not only along the axis of $0^{\circ}-$ $180^{\circ}$ or $90^{\circ}-270^{\circ}$, but also along the axis of $45^{\circ}-225^{\circ}$ or $135^{\circ}-$ $315^{\circ}$; that is, the dual-dipole antenna is four-axis reflection symmetric, it will radiate energy uniformly in the space. Theoretically, gain deviation of a dual-dipole tag antenna with four-axis reflection symmetry is approximately $3 \mathrm{~dB}$. Its schematic drawing is shown as Figure 1(a). The curves of port 1 and port 2 indicate the gain of a single port of the dualdipole and the curve of sum is total gain of the two ports. If a dual-dipole antenna is not four-axis reflection symmetric, in its null reading zone offset by each other, far-field radiation will be strengthened in some places and in some other places 


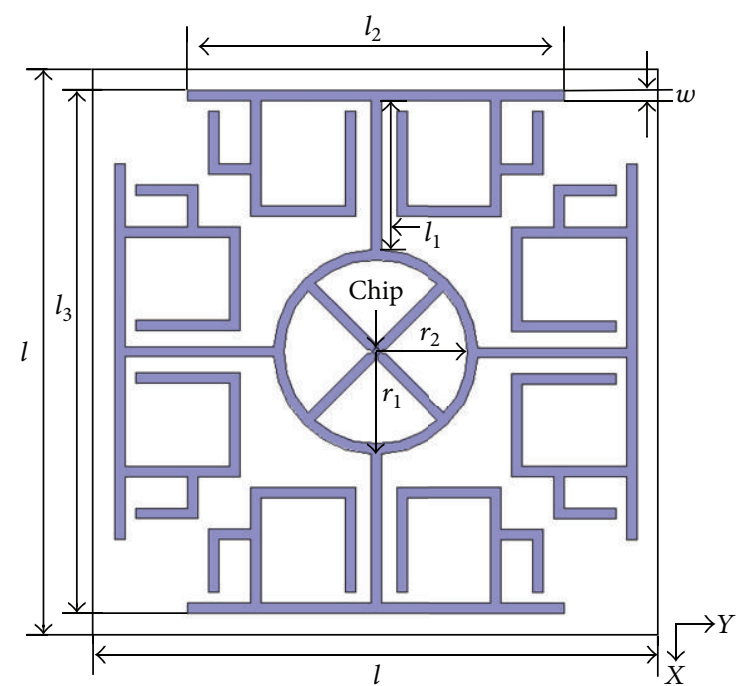

FIgURE 2: Structure of the proposed dual-dipole tag antenna. The substrate of the antenna is made of PTFE, with an area of $l \times l$ and a thickness of $h$. Main dimensions of antenna radiator are determined by $l_{1}, l_{2}, l_{3}$, and $w$, and the sizes of feed loop are determined by $r_{1}$ and $r_{2}$.

will be weakened. For this reason, the gain deviation in the entire space will be poorer than that of dual-dipole antenna with four-axis reflection symmetry. Figure 1(b) illustrates this situation.

The structure of the dual-dipole tag antenna designed in this paper is shown in Figure 2. Its center frequency is $915 \mathrm{MHz}$. The corresponding operation wavelength is about $328 \mathrm{~mm}$ in free space and is about $201 \mathrm{~mm}$ in the medium fully filled with polyfluortetraethylene (PTFE) of dielectric constant 2.65. For a half-wave dipole, its printed dipole antenna includes both medium and free space, the actual arm length of the proposed antenna should be $164-100 \mathrm{~mm}$, so average value of $132 \mathrm{~mm}$ was taken as an initial value of total length of two antenna arms, and then its actual value was given using simulation software. If the tag adopts a linear dipole, the area of dual-dipole tag is about $132 \times$ $132 \mathrm{~mm}$, which is not convenient for most cases. As long as the operation frequency is fulfilled, the tag should be as small as possible. The tag antenna is designed to be completely symmetric not only along the axis of $0^{\circ}-180^{\circ}$ or $90^{\circ}-270^{\circ}$, but also along the axis of $45^{\circ}-225^{\circ}$ or $135^{\circ}-315^{\circ}$. The two arms of the dipole are bent to fill the surrounding space as much as possible. This design can, on one hand, ensure more uniform current distribution in antenna and improve its performance of quasi-isotropic patterns, on the other hand, miniaturize the tag antenna.

\section{Simulations of the Antennas}

3.1. Simulations of Dual-Dipole Tag Antenna with Four-Axis Reflection Symmetry. The proposed antenna was simulated using HFSS (High Frequency Simulation Structure) software of Ansys Company based on the finite element method. The line width $w$ of the antenna was fixed to be $1 \mathrm{~mm}$. $l_{1}$ is a key

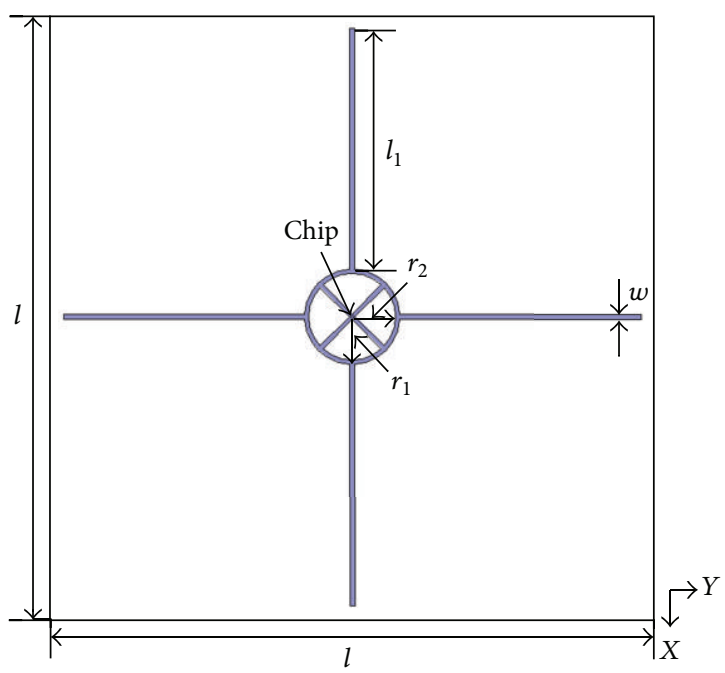

FIgURE 3: Structure of the linear dual-dipole tag antenna. The substrate of the antenna is made of PTFE, with an area of $l \times l$ and a thickness of $h$.

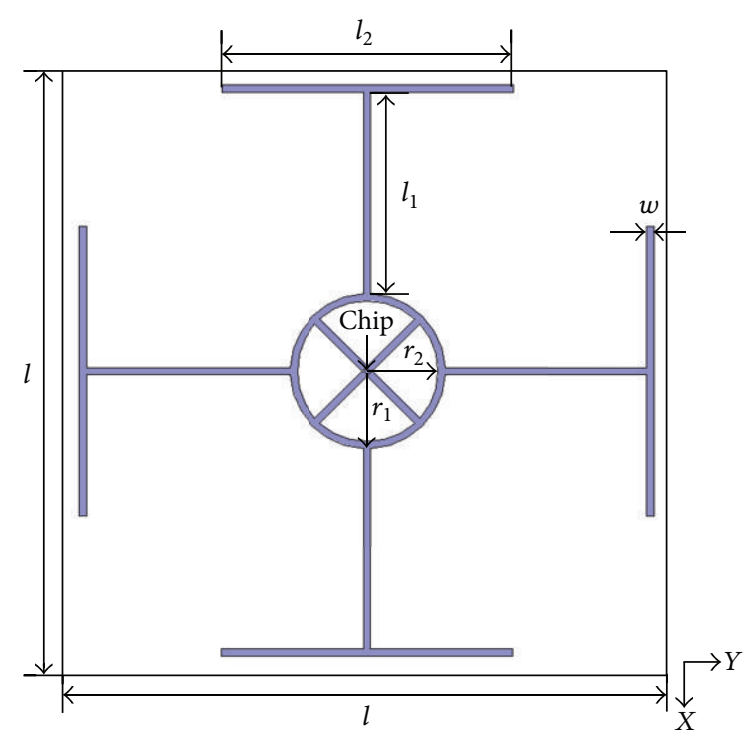

FIGURE 4: Structure of the one-fold dual-dipole tag antenna. The substrate of the antenna is made of PTFE, with an area of $l \times l$ and a thickness of $h$.

factor which determines the dimensions of the tag. During the simulation, $l_{1}$ was gradually reduced from $66 \mathrm{~mm}, l_{2}$ was increased, and the antenna was bent to fill the space between $l_{2}$ and the feed loop as much as possible. Finally its resonant frequency was adjusted to be about $915 \mathrm{MHz}$ by fine tuning $l_{2}$.

First, a linear dual-dipole tag antenna was simulated. Figure 3 shows the structure of the linear dual-dipole tag antenna. When $l_{1}$ was $55 \mathrm{~mm}$, the resonant frequency of the linear dual-dipole tag antenna can be adjusted to $914 \mathrm{MHz}$. The normalized radiation patterns of the linear dual-dipole tag antenna are shown in Figure 5. The simulated maximum and minimum gain values of the tag antenna in three planes 


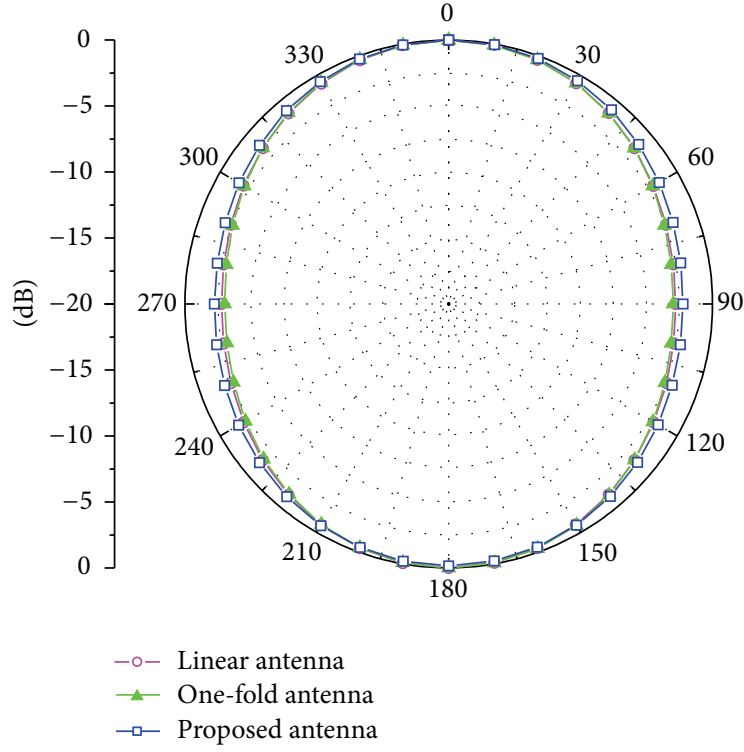

(a) $X Z$ plane

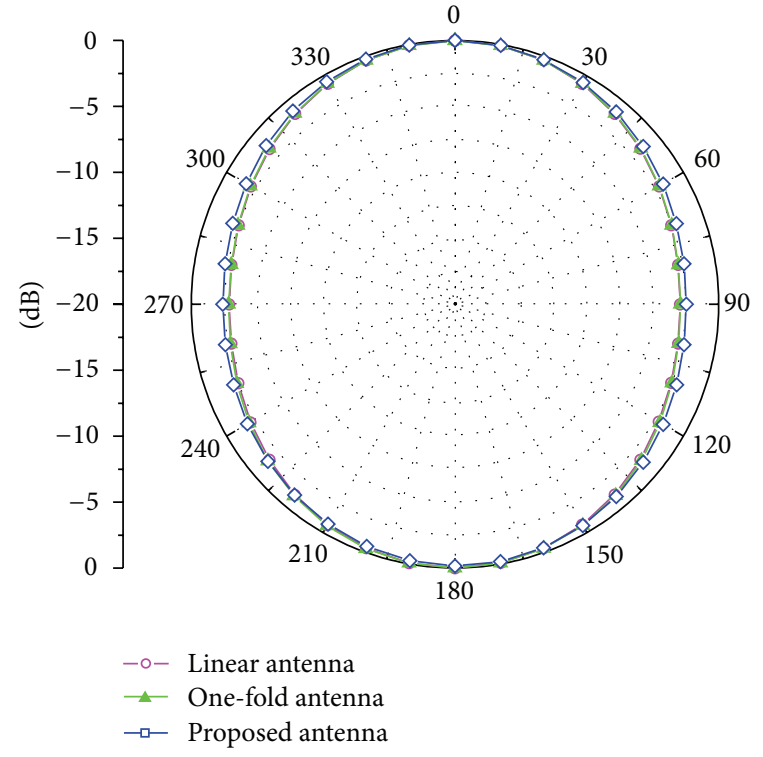

(b) YZ plane

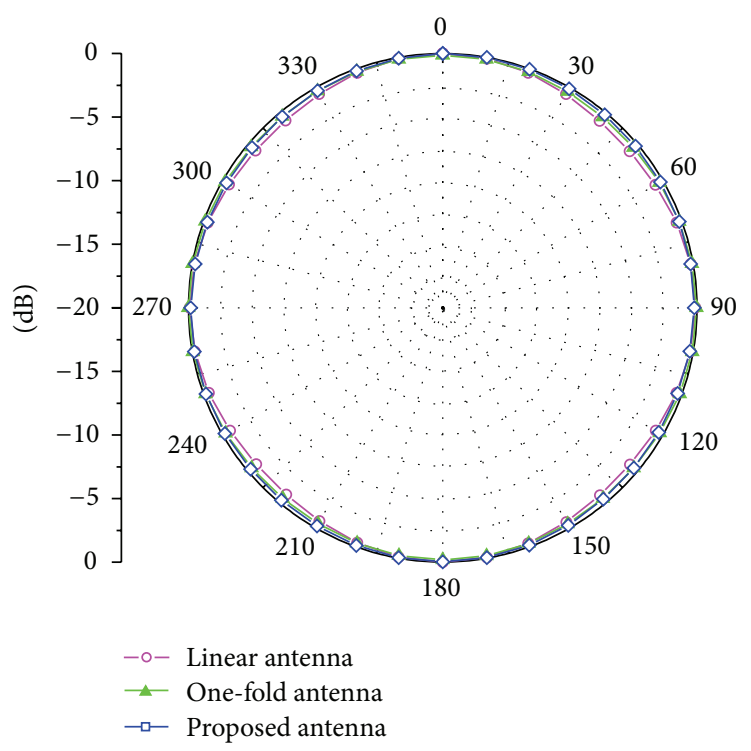

(c) $X Y$ plane

FIGURE 5: Simulated normalized radiation patterns of the three tag antennas.

TABLE 1: Simulated gain values in three planes of the three tag antennas (Unit: $\mathrm{dB}$ ).

\begin{tabular}{lcccc}
\hline Gain & $X Z$ & $Y Z$ & $X Y$ & Gain deviation \\
\hline Linear antenna max. & 5.08 & 5.08 & 2.29 & \multirow{2}{*}{3.64} \\
Linear antenna min. & 2.28 & 2.15 & 1.44 & \\
One-fold antenna max. & 4.98 & 4.98 & 2.13 & 3.28 \\
One-fold antenna min. & 1.96 & 2.08 & 1.70 & \\
Proposed antenna max. & 4.51 & 4.51 & 2.28 & 2.64 \\
Proposed antenna min. & 2.26 & 2.05 & 1.87 &
\end{tabular}

are listed in Table 1, with a gain deviation of $3.64 \mathrm{~dB}$ in different directions.
Then the segment $l_{2}$ was added on the linear dual-dipole tag antenna and the segment $l_{1}$ was gradually shortened. The linear dual-dipole tag antenna became a one-fold dual-dipole tag antenna and the structure of the one-fold dual-dipole tag antenna is shown as Figure 4 . When $l_{1}$ and $l_{2}$ were $28 \mathrm{~mm}$ and $40 \mathrm{~mm}$, respectively, the resonant frequency of the onefold dual-dipole tag antenna can be adjusted to $916 \mathrm{MHz}$. The normalized radiation patterns of the one-fold dual-dipole tag antenna are shown in Figure 5. Table 1 illustrates the maximum and minimum gain values of the tag antenna in three planes, with a gain deviation of $3.28 \mathrm{~dB}$ in different directions.

In order to obtain the uniform radiation and reduce the area of tag antenna, the antenna was bent to fill the space 
TABLE 2: Parameter values of optimized antenna.

\begin{tabular}{lccccccc}
\hline Parameters & $l$ & $l_{1}$ & $l_{2}$ & $l_{3}$ & $w$ & $r_{1}$ & $r_{2}$ \\
\hline Values $(\mathrm{mm})$ & 54.0 & 15.0 & 18.0 & 50.0 & 1.0 & 9.8 & 8.8 \\
\hline
\end{tabular}

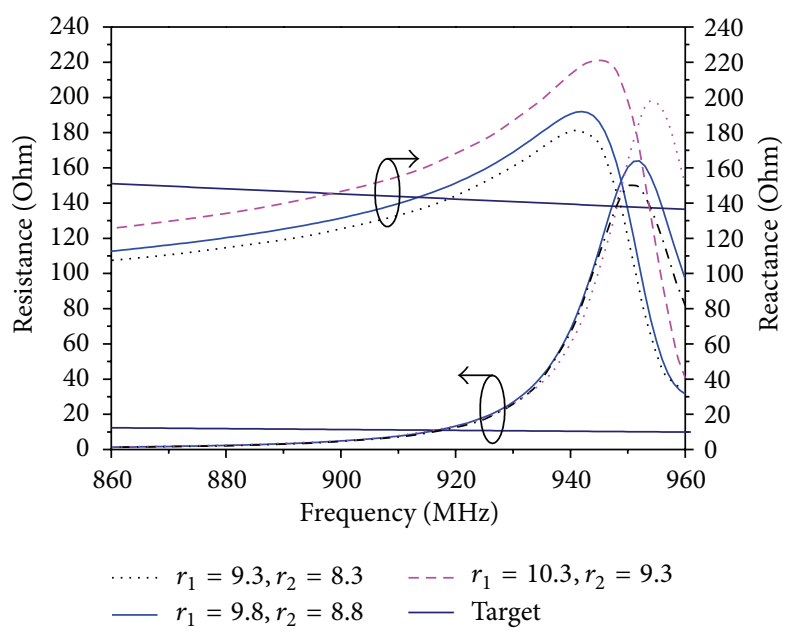

FIGURE 6: Simulated impedance of the proposed antenna with different sizes of the feed loop.

between $l_{2}$ and the feed loop while the antenna became a multi-fold dual-dipole tag antenna, as shown in Figure 2. If the main parameters of the tag antenna are set following Table 2, its resonant frequency can be adjusted to $914 \mathrm{MHz}$. The normalized radiation patterns of the tag antenna are shown in Figure 5. The maximum and minimum gain values of the tag antenna in three planes are listed in Table 1, with a gain deviation of $2.64 \mathrm{~dB}$ in different directions.

The above simulated results show that the quasi-isotropic performance of tag antenna is easier to be obtained as long as the dual-dipole tag antenna is four-axis reflection symmetric. As the dipole arms are bent to fill the antenna space gradually, the quasi-isotropic performance of tag antenna becomes better since the gain deviation of the antenna radiation pattern reduces gradually.

Impedance match of the antenna can be achieved mainly through the adjustment of sizes of feed loop if the four arms of dual-dipole are basically constant. The width of feed loop was fixed to be $1 \mathrm{~mm}$, and by adjusting its inner radius and outer radius, appropriate impedance can be obtained to match the impedance of chip. The Monza $4 \mathrm{D}$ chip from Impinj was used, with an impedance of $11-j 143 \Omega$ at $915 \mathrm{MHz}$ [9]. Figure 6 illustrates the simulated impedance curves of the antenna with different sizes of the feed loop. Figure 7 shows the simulated reflection coefficient curves of the antenna with different sizes of the feed loop. It can be seen from Figures 6 and 7 that if outer radius of feed loop is $9.3 \mathrm{~mm}$ and its inner radius is $8.3 \mathrm{~mm}$, the impedance of the antenna matches chip best and the reflection coefficient $S_{11}$ is $-30.07 \mathrm{~dB}$. Considering the resonant frequency, its outer radius and inner radius were eventually selected to be $9.8 \mathrm{~mm}$ and $8.8 \mathrm{~mm}$, respectively. The thick solid line parts of Figures 6 and 7 are the corresponding curves.

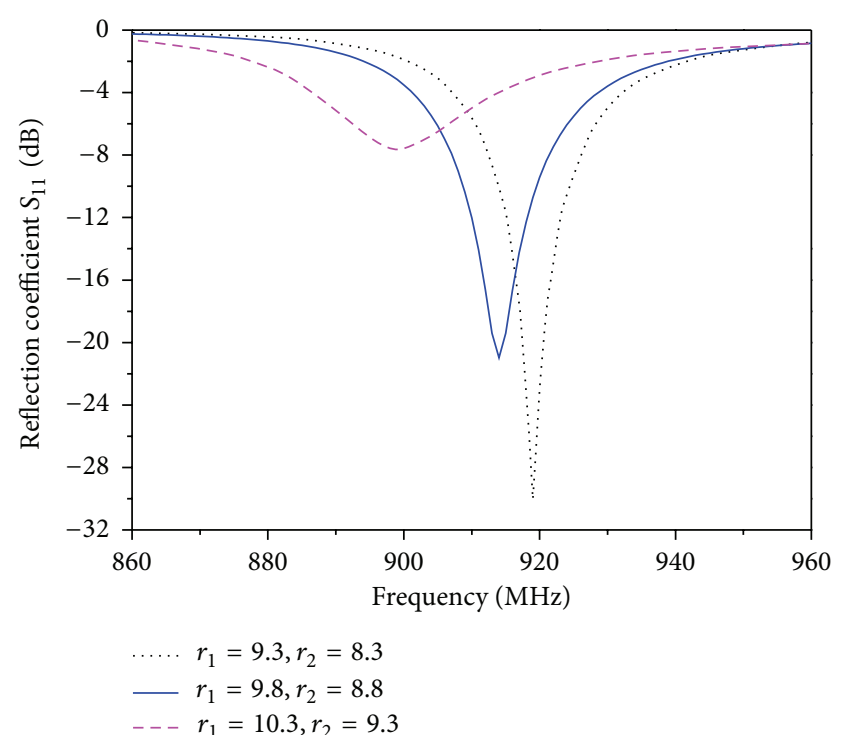

FIGURE 7: Simulated reflection coefficient of the proposed antenna with different sizes of the feed loop.

TABLE 3: Dimensions of the three commercial tag antennas.

\begin{tabular}{llllccc}
\hline Tags & \multicolumn{2}{c}{ Invengo TF8023 } & \multicolumn{2}{c}{ Impinj H47 } & \multicolumn{2}{c}{ UPM frog 3D } \\
\hline Parameters & $L$ & $L_{1}$ & $L$ & $L_{1}$ & $L$ & $L_{1}$ \\
Values $(\mathrm{mm})$ & 65 & 70 & 54 & 44 & 54 & 50 \\
\hline
\end{tabular}

TABLE 4: Simulated gain values of the three commercial tag antennas in three planes (Unit: $\mathrm{dB}$ ).

\begin{tabular}{lcccc}
\hline Gain & $X Z$ & $Y Z$ & $X Y$ & $\begin{array}{c}\text { Gain } \\
\text { deviation }\end{array}$ \\
\hline Invengo TF8023 max. & 4.89 & 4.89 & 1.97 & 4.02 \\
Invengo TF8023 min. & 1.94 & 1.00 & 0.87 & \\
Impinj H47 max. & 4.83 & 4.83 & 1.59 & 4.39 \\
Impinj H47 min. & 1.56 & 1.41 & 0.44 & \\
UPM frog 3D max. & 4.87 & 4.87 & 2.61 & 5.12 \\
UPM frog 3D min. & 1.17 & 1.40 & -0.25 & \\
\hline
\end{tabular}

It can be seen in Figure 7 that the reflection coefficient $S_{11}$ is $-20.97 \mathrm{~dB}$ at the resonance frequency of $914 \mathrm{MHz}$. All parameter values of optimized antenna are listed in Table 2.

3.2. Compare with Three Commercial Quasi-Isotropic Tag Antennas. For comparison, three representative commercial quasi-isotropic tags, Invengo TF8023, Impinj H47, and UPM frog $3 \mathrm{D}$, were also simulated. The three tags are all designed with Monza 4 of Impinj. Figure 8 shows the structures of the above three tag antennas. It can be seen from the figure that these antennas use non-four-axis reflection symmetric structures. Main dimensions of the antennas are listed in Table 3. And the simulated maximum and minimum gain values of the tag antennas in three planes are listed in Table 4, 


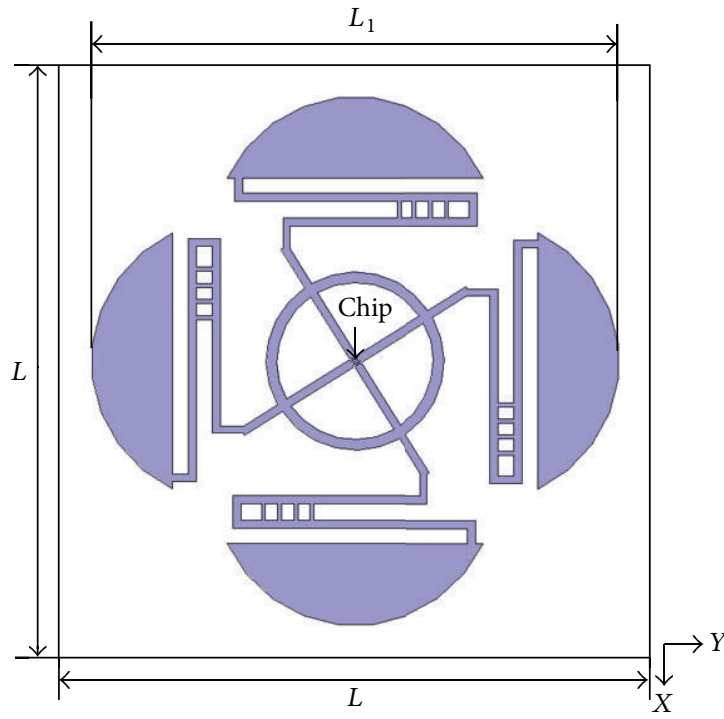

(a) Invengo TF8023

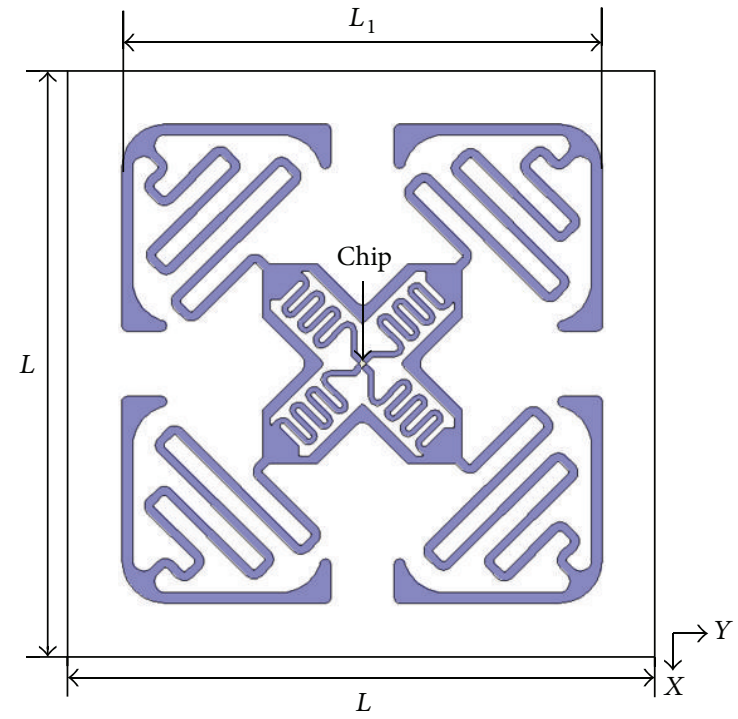

(b) Impinj $\mathrm{H} 47$

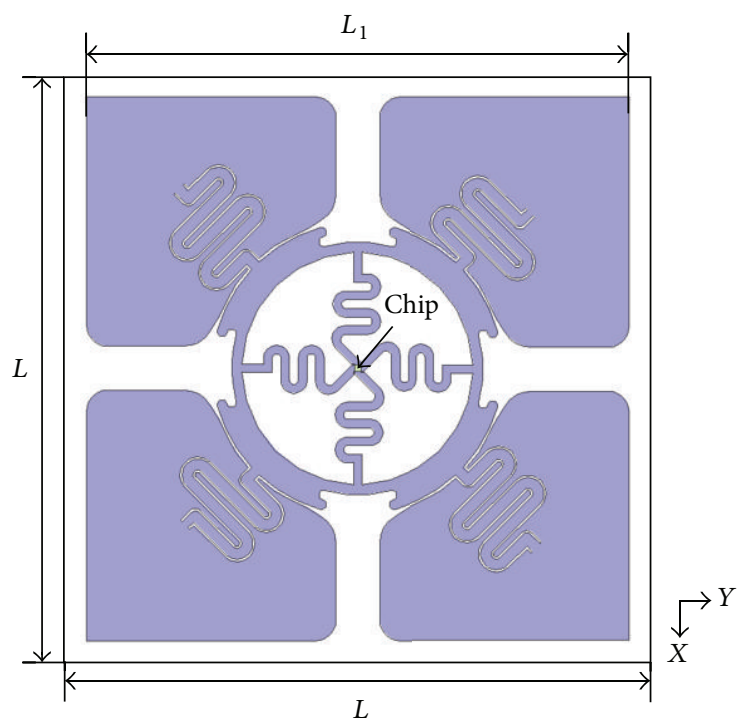

(c) UPM frog 3D

FIGURE 8: Structure of the three commercial tag antennas. The area of substrate is $L \times L$, the material of substrate is PET, and the area of antenna radiator is $L_{1} \times L_{1}$.

with a gain deviation of $4.02 \mathrm{~dB}$ for Invengo TF8023, $4.39 \mathrm{~dB}$ for Impinj H47, and $5.12 \mathrm{~dB}$ for UPM Frog 3D.

The simulation results show that the tag antenna with four-axis reflection symmetric structure, due to its uniform far-field radiation, has less gain deviation than a tag antenna with non-four-axis reflection symmetric structure.

\section{Measurement and Analysis}

The photograph of the fabricated dual-dipole tag designed in this paper is shown in Figure 9. The PTFE substrate was used for the tag, with the parameters of dielectric constant 2.65,

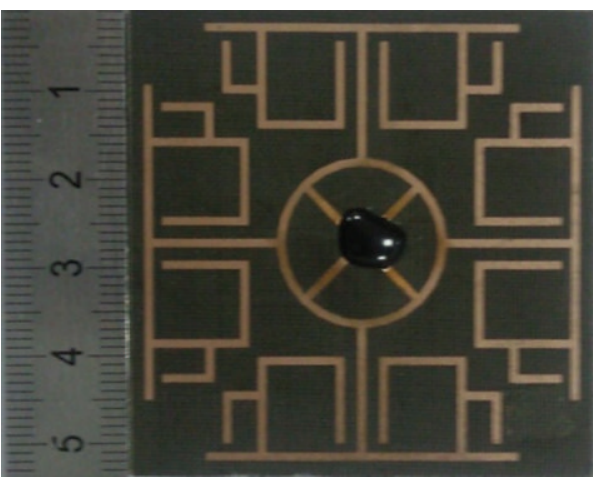

FIgURE 9: Photograph of the fabricated tag. 
loss tangent 0.002 , and thickness $0.765 \mathrm{~mm}$. And the tag chip was connected to the antenna by bonding.

To verify the performance of the tag, its reading orientation, reading range, and antenna impedance were tested. For comparison, Invengo TF8023, Impinj H47, and UPM frog 3D were also tested. Speedway Revolution Reader of Impinj, with operation frequency of $902 \mathrm{MHz}-928 \mathrm{MHz}$, output power of $10 \mathrm{dBm}-30 \mathrm{dBm}$, and adjustment step of $0.25 \mathrm{dBm}$, was used to test reading orientation and reading range. CSL-RHCP of CSL Company was used as a reader antenna, with gain of $6 \mathrm{dBi}$.

The reading range and the reading orientation of the tags were measured in an anechoic chamber as illustrated in Figure 10. In the anechoic chamber, the tag is placed at $d$ $(d=1 \mathrm{~m})$ distance from the reader antenna. The minimum power $P_{\min }$ required to communicate with the tag is obtained by varying the reader output power. The reading range $r$ can be calculated using Friis free-space deformational formula as [10]

$$
r=d \sqrt{\frac{\mathrm{EIRP}}{P_{\min } L G_{t}}}
$$

where EIRP is effective isotropic radiated power, $L$ is the loss of the connecting cable, and $G_{t}$ is the gain of the reader antenna.

According to the parameter test method of performance of EPC UHF RFID tag [11], the tag was tested in three different planes with a measurement point every $10^{\circ}$. The gain values of the four tags in each direction were normalized with a maximum value of $0 \mathrm{~dB}$. The measured radiation patterns are illustrated in Figure 11. The maximum and minimum values of power required for the test of four tag antennas in three planes are listed in Table 5. From the table, the tag antenna designed in this paper has a gain deviation of $3.25 \mathrm{~dB}$, while gain deviations of tag antennas of Invengo TF8023, Impinj $\mathrm{H} 47$, and $\mathrm{UPM}$ frog $3 \mathrm{D}$ are $5.25 \mathrm{~dB}, 5.75 \mathrm{~dB}$, and $6.5 \mathrm{~dB}$, respectively. The tag designed in this paper has a maximum reading range of 6.9-10.0 meters in different directions at the frequency of $915 \mathrm{MHz}$, while the value is $4.4-8.0$ meters for Invengo TF8023, 4.2-8.2 meters for Impinj H47, and 3.1-6.5 meters for UPM frog $3 \mathrm{D}$.

The test results indicate that the tag antenna designed in this paper, due to its four-axis reflection symmetry, has better quasi-isotropic performance than a tag antenna with nonfour-axis reflection symmetric structure.

MS4624B vector network analyzer of Anritsu Company was adopted to measure the impedance of the tag antenna. The dual-dipole antenna designed in this paper can be considered as two dipole antennas with single port. Dipole antenna is a kind of balanced antenna and cannot be directly measured with a vector network analyzer. According to analysis of Tikhov et al., indirect measurement based on the method of images is feasible [12]. Referring to the method introduced by Tikhov et al. [12], a setup was designed for the measurement of the antenna impedance. The structure of the measurement setup is shown in Figure 12. It is composed of three parts: the metal plate, a half of the antenna (the monopole antenna), and an SMA connector. An infinite

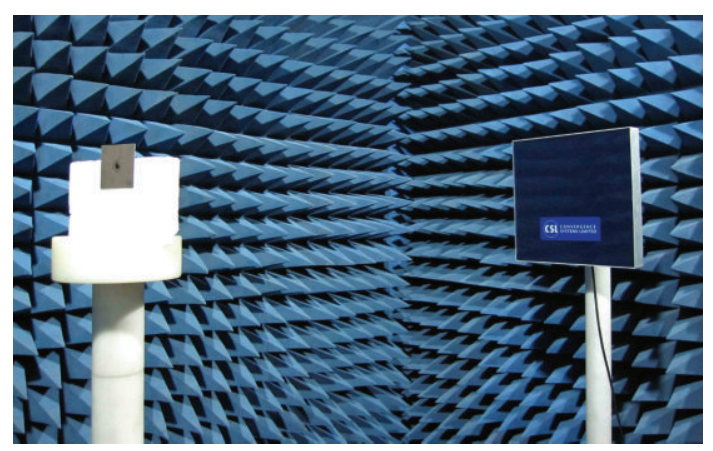

FIgURE 10: Measurement environment of the tag.

TABLE 5: Minimum power required for the test of the four kinds of tag antennas in three planes.

\begin{tabular}{lcccc}
\hline Power & $\begin{array}{c}X Z \\
(\mathrm{dBm})\end{array}$ & $\begin{array}{c}Y Z \\
(\mathrm{dBm})\end{array}$ & $\begin{array}{c}X Y \\
(\mathrm{dBm})\end{array}$ & $\begin{array}{c}\text { Gain deviation } \\
(\mathrm{dB})\end{array}$ \\
\hline Proposed antenna max. & 14.00 & 13.75 & 14.75 & 3.25 \\
Proposed antenna min. & 11.75 & 11.50 & 13.25 & \\
Invengo TF8023 max. & 15.00 & 15.25 & 18.75 & 5.25 \\
Invengo TF8023 min. & 14.00 & 13.50 & 16.00 & \\
Impinj H47 max. & 15.75 & 17.25 & 19.00 & 5.75 \\
Impinj H47 min. & 13.25 & 13.50 & 15.75 & \\
UPM frog 3D max. & 17.25 & 17.25 & 21.75 & 6.50 \\
UPM frog 3D min. & 15.25 & 15.50 & 19.50 & \\
\hline
\end{tabular}

plane is simulated by the metal plate which consists of a stainless steel part $(700 \times 700 \mathrm{~mm})$ and a copper part $(160$ $\times 160 \mathrm{~mm}$ ). The monopole antenna is placed on the copper plate which can weld the antenna conveniently. The SMA connector is hidden under the copper plate. While measuring, the SMA connector of the vector network analyzer was connected to the SMA connector of the measurement setup. The vector network analyzer fed to the antenna and measured the impedance of the monopole antenna. The input impedance of the dipole tag antenna was twice of the measured impedance of the monopole antenna. As the vector network analyzer can be only precalibrated to the port output terminal, increased phase change due to SMA connector was corrected through simulation and calculation. The measured antenna impedance was obtained, as shown in Figure 13. It can be seen that the real part and the imaginary part of the measured antenna impedance intersect with the target values at about $915 \mathrm{MHz}$.

Using a complex impedance reflection coefficient calculation formula, the measured power reflection coefficient curve was converted with the method described by Mo and Qin [13]. Figure 14 shows the measured power reflection coefficient of the antenna. It can be seen from the figure that the measured resonant frequency of the antenna is $921 \mathrm{MHz}$ and its reflection coefficient $S_{11}$ is $-25.3 \mathrm{~dB}$.

\section{Conclusion}

In this paper, a passive UHF dual-dipole tag antenna with quasi-isotropic patterns is designed, which can be read 


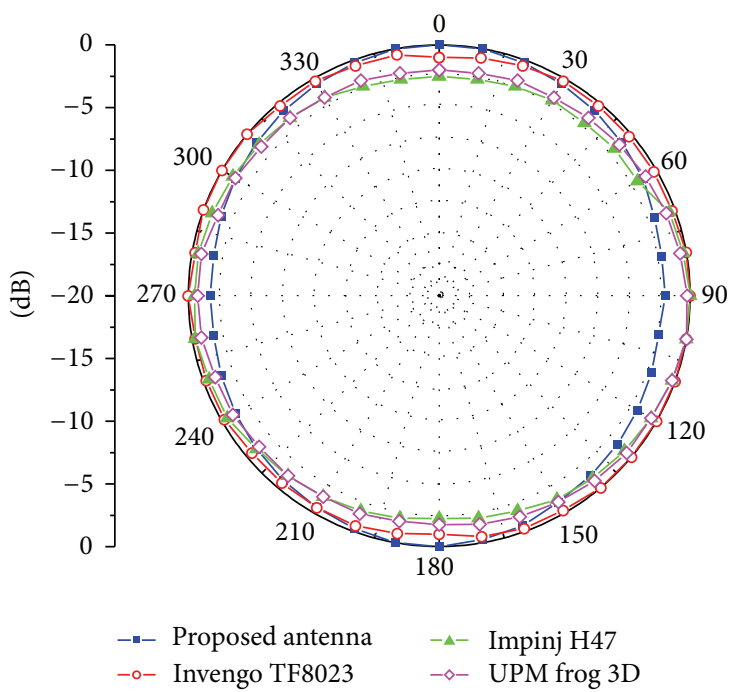

(a) $X Z$ plane

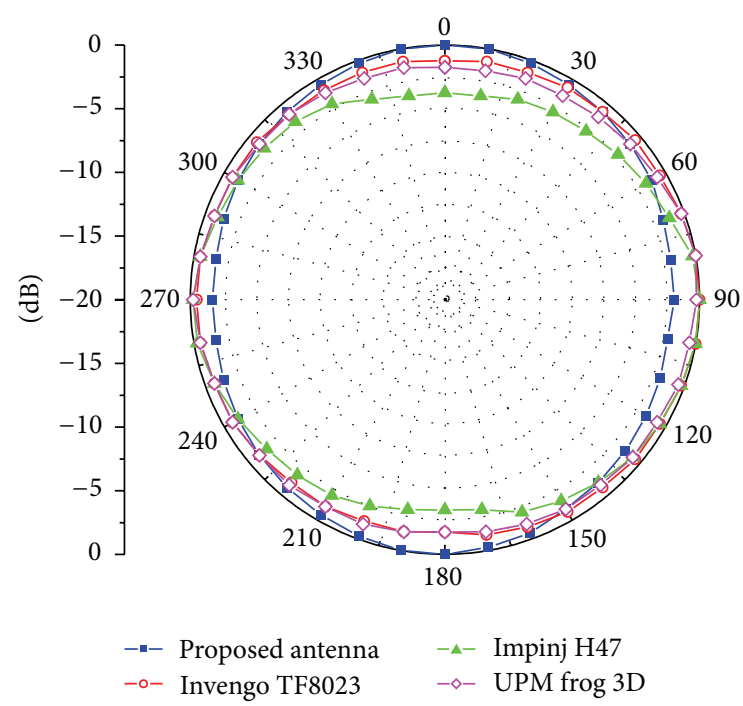

(b) YZ plane

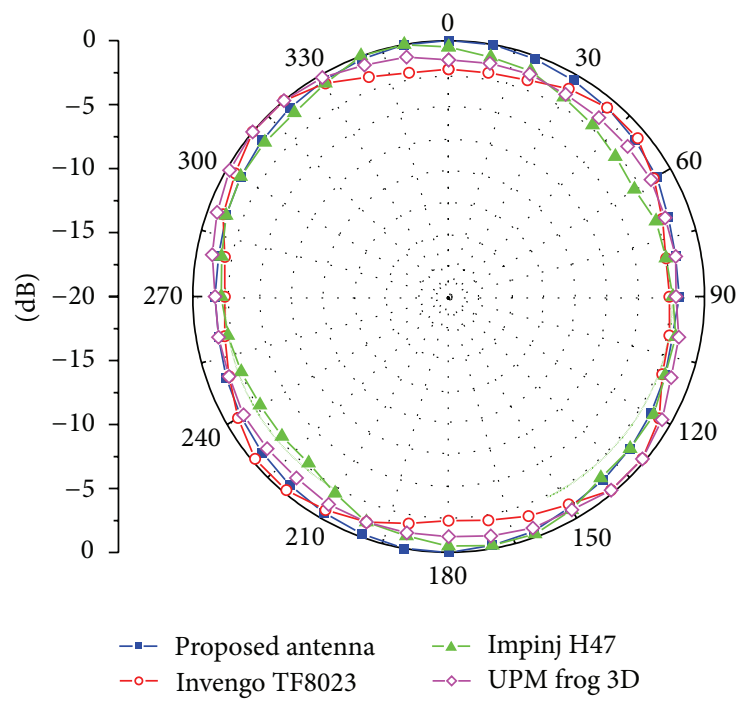

(c) $X Y$ plane

FIGURE 11: Measured normalized radiation patterns of the four kinds of tag antennas.

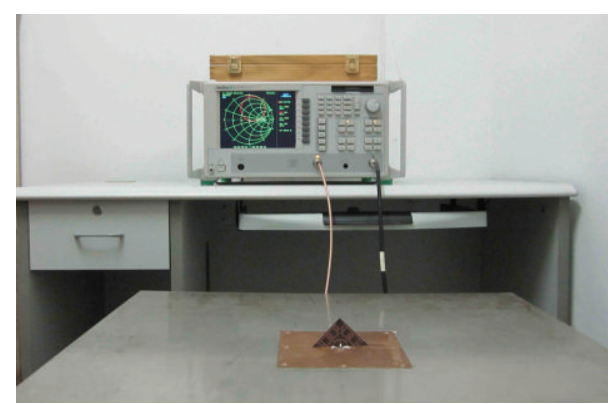

Figure 12: Measurement setup for the proposed antenna impedance.

reliably from a long distance in different directions. Owing to the four-axis reflection symmetric structure and the bent arms, its gain deviation in any arbitrary direction is approximately $3 \mathrm{~dB}$ and it could be reliably read within a range of more than 6 meters in different directions. Three representative commercial dual-dipole quasi-isotropic tag antennas were compared in this paper. The results show that the proposed four-axis reflection symmetric tag antenna is with better quasi-isotropic performance and reading range than other commercial tags. Therefore, to effectively reduce null reading zone of ordinary single dipole and realize a larger reading range, four-axis reflection symmetric structure should be used for dual-dipole tag antenna with quasiisotropic patterns.

\section{Conflict of Interests}

The authors do not have any conflict of interests with the content of the paper. 


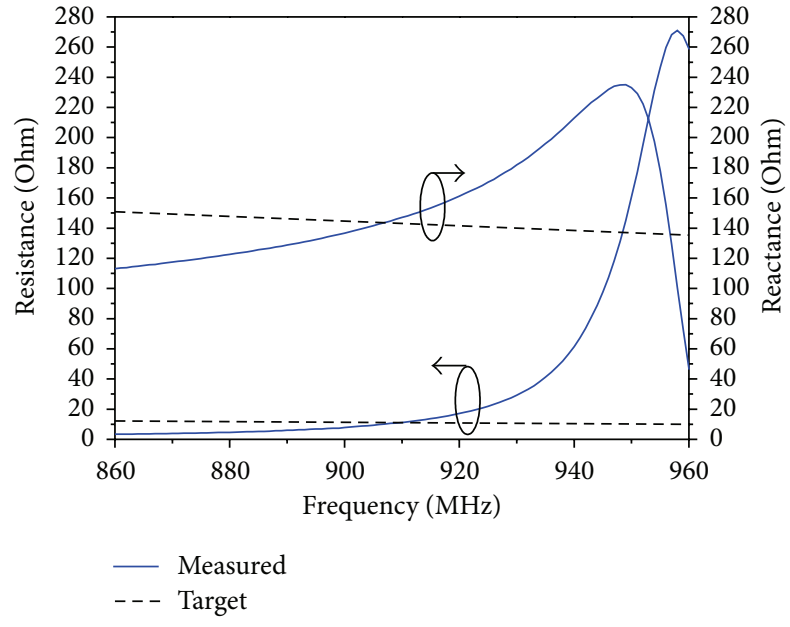

Figure 13: Measured impedance of the proposed antenna.

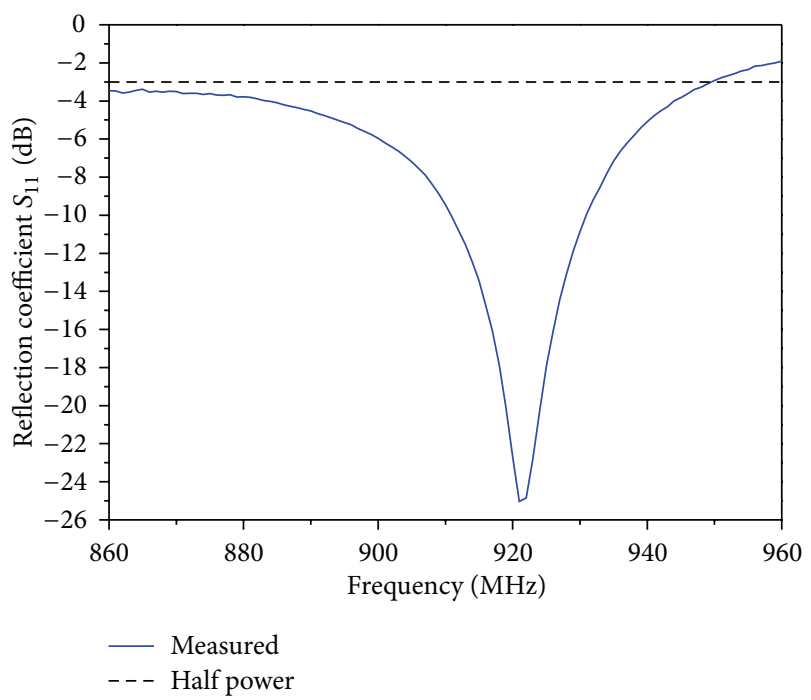

FIGURE 14: Measured reflection coefficient of the proposed antenna.

\section{Acknowledgments}

This work was supported by the National Key Technology R\&D Program of China (2009BADB9B09) and the National Water Pollution Control and Management Technology Major Projects (2008ZX07420-004).

\section{References}

[1] C. Cho, H. Choo, and I. Park, "Broadband RFID tag antenna with quasi-isotropic radiation pattern," Electronics Letters, vol. 41, no. 20, pp. 1091-1092, 2005.

[2] D. V. Kholodnyak, P. A. Turalchuk, A. B. Mikhailov, S. Y. Dudnikov, and I. B. Vendik, "3D antenna for UHF RFID tags with eliminated read-orientation sensitivity," in Proceedings of the 36th European Microwave Conference (EuMC '06), vol. 1-4, pp. 583-586, Manchester, UK, September 2006.

[3] H. W. Wang, Y. L. Wang, Y. Liu, and W. S. Yu, "3D antenna for UHF RFID tags with near omni-direction," in Proceedings of the 8th International Symposium on Antennas, Propagation and EM Theory (ISAPE '08), vol. 1-3, pp. 118-121, Kunming, China, November 2008.

[4] J. Ahn, H. M. Jang, H. S. Moon, J. W. Lee, and B. Lee, "Inductively coupled compact RFID tag antenna at $910 \mathrm{MHz}$ with nearisotropic radar cross-Section (RCS) patterns," IEEE Antennas and Wireless Propagation Letters, vol. 6, pp. 518-520, 2007.

[5] C. Cho, H. Choo, and I. Park, "Printed symmetric inverted$\mathrm{f}$ antenna with a quasi-isotropic radiation pattern," Microwave and Optical Technology Letters, vol. 50, no. 4, pp. 927-930, 2008.

[6] S. L. Chen, K. H. Lin, and R. Mittra, "Miniature and near-3D omnidirectional radiation pattern RFID tag antenna design," Electronics Letters, vol. 45, no. 18, pp. 923-924, 2009.

[7] H. W. Son and C. S. Pyo, "Design of RFID tag antennas using an inductively coupled feed," Electronics Letters, vol. 41, no. 18, pp. 994-996, 2005.

[8] C. F. Qin, L. F. Mo, H. L. Zhou, and H. J. Zhang, "A single port dipole for UHF RFID tag antennas with eliminated readorientation sensitivity," in Proceedings of the International Conference on Consumer Electronics, Communications and Networks (CECNet '11), pp. 998-1001, Xianning, China, April 2011.

[9] "Monza 4 Tag Chip Datasheet," http://www.impinj.com/.

[10] K. V. S. Rao, P. V. Nikitin, and S. F. Lam, "Antenna design for UHF RFID tags: a review and a practical application," IEEE Transactions on Antennas and Propagation, vol. 53, no. 12, pp. 3870-3876, 2005.

[11] EPCglobal Inc., "Tag Performance Parameters and Test Methods," 2008.

[12] Y. Tikhov, Y. Kim, and Y. H. Min, "A novel small antenna for passive RFID transponder," in Proceedings of the 35th European Microwave Conference, vol. 1-3, pp. 257-260, Paris, France, October 2005.

[13] L. F. Mo and C. F. Qin, "Tunable compact UHF RFID metal tag based on CPW open stub feed PIFA antenna," International Journal of Antennas and Propagation, vol. 2012, Article ID 167658, 8 pages, 2012. 

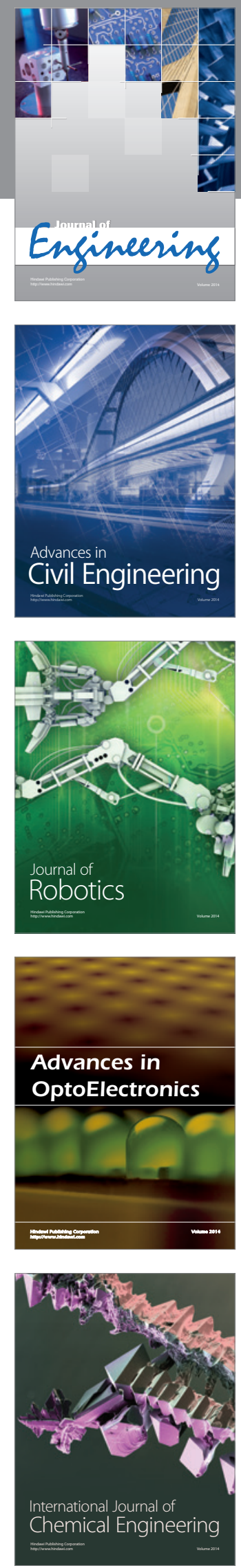

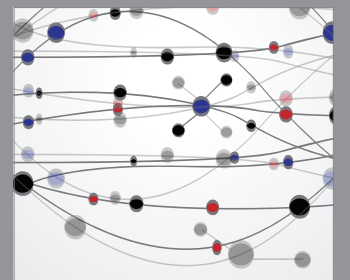

The Scientific World Journal
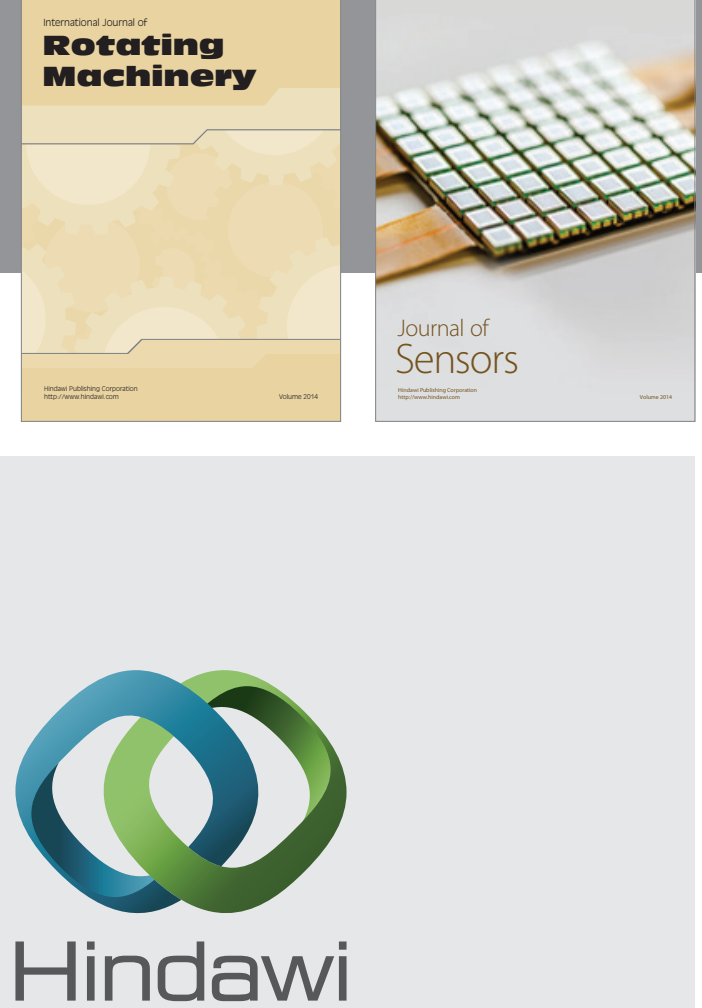

Submit your manuscripts at http://www.hindawi.com
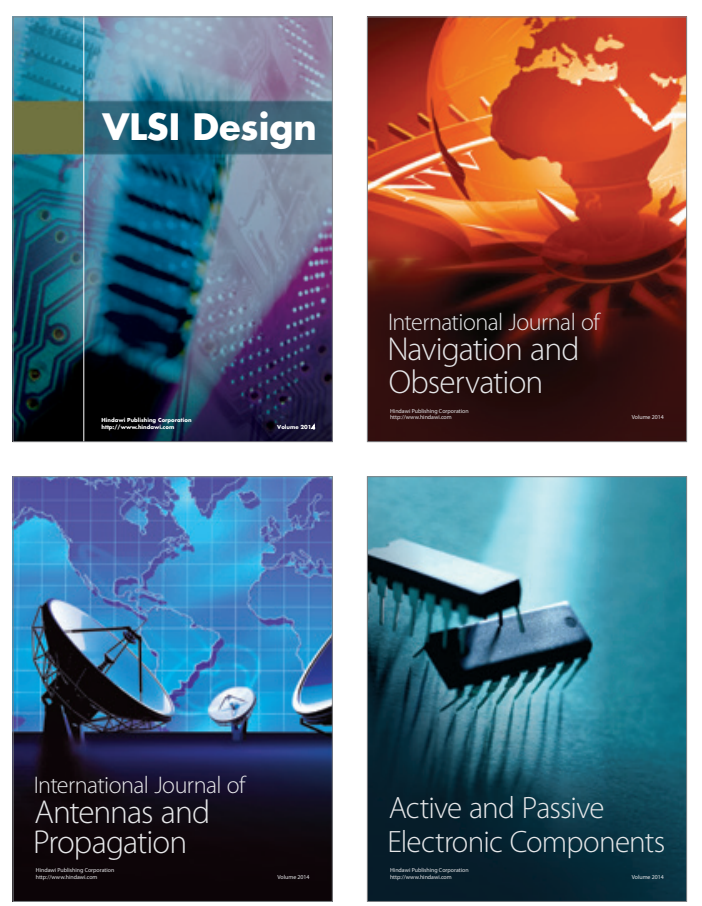
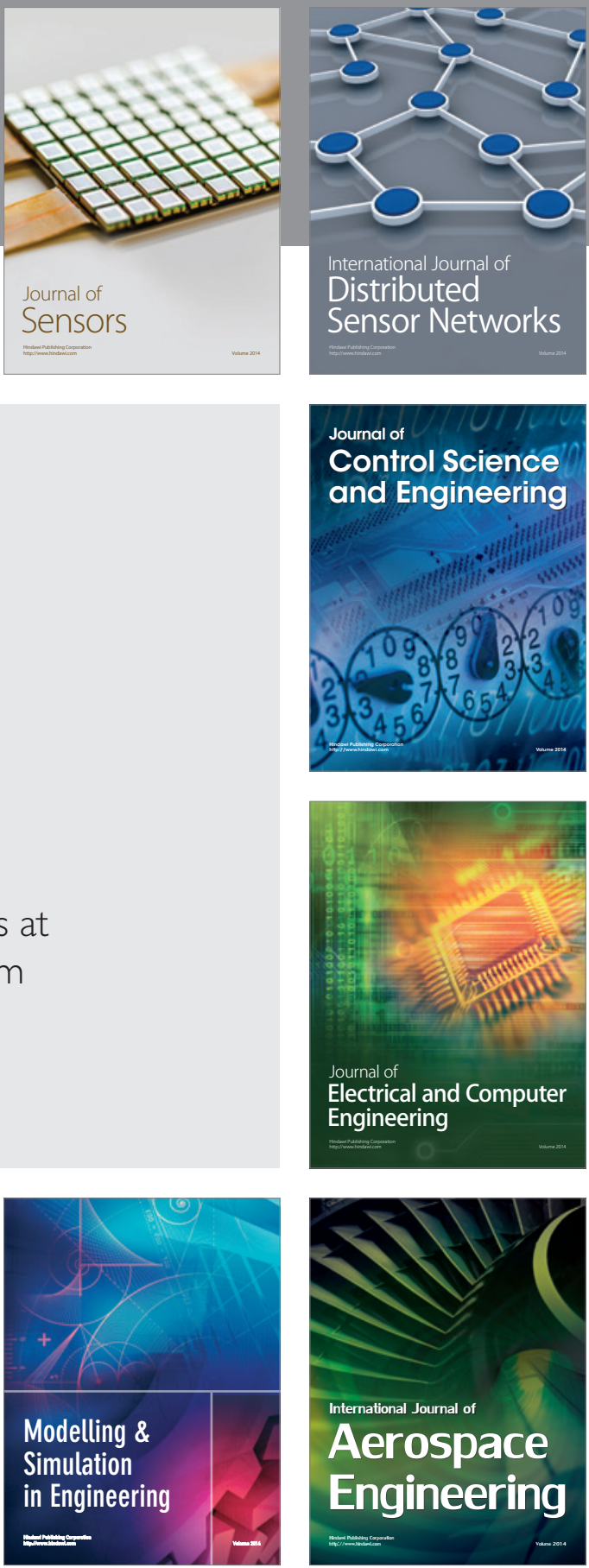

Journal of

Control Science

and Engineering
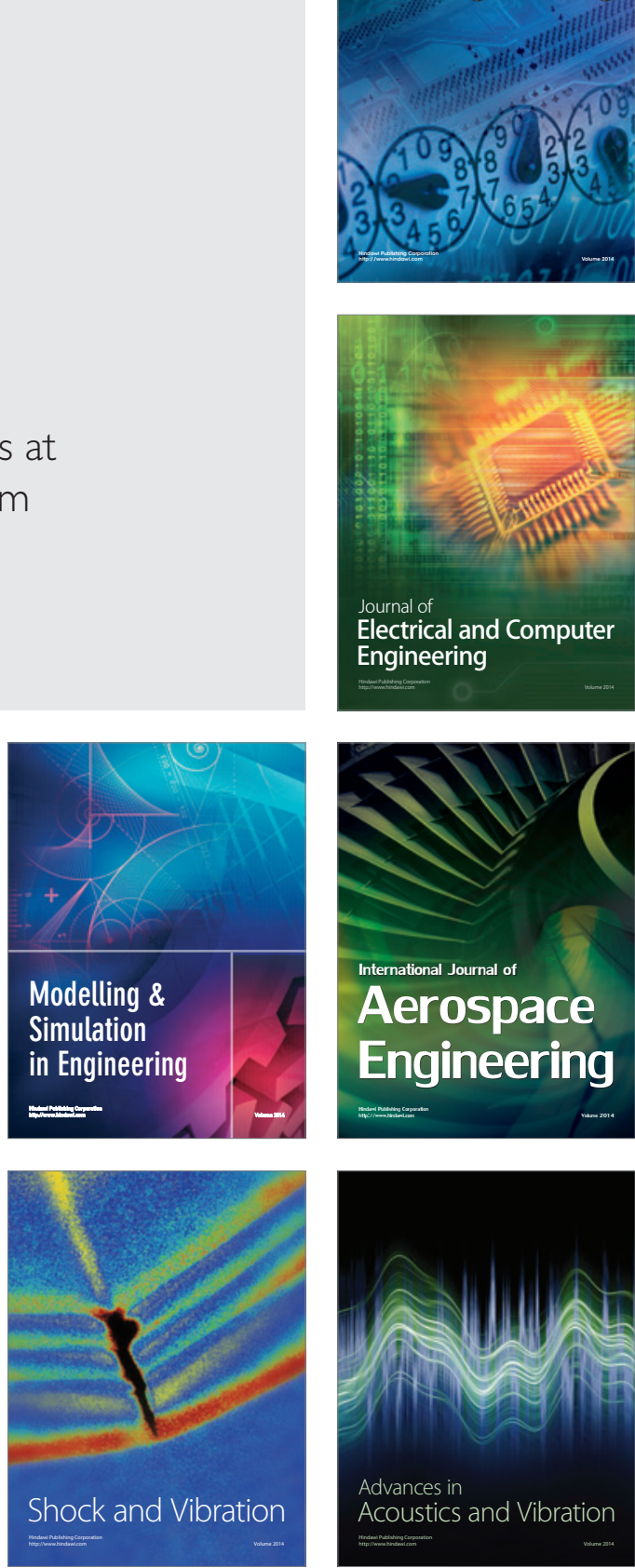